\title{
Non-radiative inelastic processes in lithium-helium ion-atom collisions
}

\author{
Andrey K. Belyaev ${ }^{1}$, Lucie Augustovičová ${ }^{2}$, Pavel Soldán ${ }^{2}$, and Wolfgang P. Kraemer ${ }^{3}$ \\ 1 Department of Theoretical Physics and Astronomy, Herzen University, St. Petersburg 191186, Russia and Max-Planck Institute for \\ Astrophysics, Postfach 1371, 85741 Garching, Germany \\ e-mail: andrey.k.belyaev@gmail.com \\ 2 Charles University in Prague, Faculty of Mathematics and Physics, Department of Chemical Physics and Optics, Ke Karlovu 3 , \\ 12116 Prague 2, Czech Republic \\ 3 Max-Planck Institute for Astrophysics, Postfach 1371, 85741 Garching, Germany
}

Received 5 February 2014 / Accepted 12 March 2014

\section{ABSTRACT}

\begin{abstract}
Aims. The aims are to estimate efficiencies of non-radiative inelastic processes in lithium-helium ion-atom collisions and to compare them to those for radiative processes.

Methods. Non-radiative inelastic cross-sections and rate coefficients for different lithium-helium ion-atom collisions are estimated by means of the recently proposed branching probability current method, which is based on the accurate ab initio adiabatic BornOppenheimer potentials that have been recently calculated for the low-lying ${ }^{1,3} \Sigma^{+}$and ${ }^{1,3} \Pi$ states of the $\mathrm{LiHe}^{+}$ion.

Results. It is shown that at low temperatures the radiative depopulation in $\mathrm{Li}^{+}+\mathrm{He}$ and $\mathrm{Li}+\mathrm{He}^{+}$collisions dominates over the non-radiative processes, while in $\mathrm{Li}^{+}+\mathrm{He}\left(2 \mathrm{~s}{ }^{3} \mathrm{~S}\right)$ collisions the non-radiative processes dominate over the radiative association at temperatures above $3000 \mathrm{~K}$, which can be expected to have some influence on depopulations of metastable He in high temperature astrophysical environments.
\end{abstract}

Key words. atomic data - molecular data - scattering

\section{Introduction}

Studies of non-adiabatic effects in the interactions between $\left(\mathrm{Li}^{+}+\mathrm{He}\right)$ and $\left(\mathrm{Li}+\mathrm{He}^{+}\right)$collisional complexes on different excitation levels can contribute to a better understanding of primordial and interstellar lithium chemistry, especially when the efficiencies of radiative and non-radiative processes at different temperatures are compared.

According to the Big Bang nucleosynthesis theory, lithium is the heaviest stable element produced in this event (Burles et al. 2001). After the initial period, when in the rapidly expanding Universe matter and radiation were in close thermal contact, the Universe became transparent and temperature was sufficiently lowered to allow the initially formed fully stripped atomic nuclei $\left(\mathrm{H}^{+}, \mathrm{D}^{+}, \mathrm{He}^{2+}, \mathrm{Li}^{3+}\right)$ to recombine with electrons to form finally neutral atoms. In the case of lithium, the recombination process proceeded sequentially in the order of its ionization potentials: beginning with $\mathrm{Li}^{3+}$, ending with both $\mathrm{Li}$ atoms and remaining $\mathrm{Li}^{+}$ions, which could not readily recombine due to a lack of free electrons by the end of the recombination era. Intensive studies were performed to investigate the primordial lithium chemistry (Stancil \& Zygelman 1996; Stancil \& Dalgarno 1997; Galli \& Palla 1998, 2013) and to analyze the effect of neutral lithium on the cosmic microwave background anisotropies (Palla et al. 1995; Loeb 2001). At the time when the fractional abundances of $\mathrm{Li}$ and $\mathrm{Li}^{+}$became comparable at a $z \approx 100$ redshift (Stancil et al. 2002), the abundance of $\mathrm{He}^{+}$ions dropped by several orders of magnitude (Stancil et al. 1998). At smaller redshifts, reactions of neutral $\mathrm{Li}$ atoms with $\mathrm{He}^{+}$ions are therefore rather improbable. They are, however, included in this study because $\left(\mathrm{Li}+\mathrm{He}^{+}\right)$collision pairs are obtained here via charge transfer processes in $\left(\mathrm{Li}^{+}+\mathrm{He}\right)$ collisions. In addition, even if lithium is rather rare compared to the other primordial elements synthesized in the early Universe, inelastic collisional processes of neutral lithium with hydrogen and other atomic or ionic species are of high interest in modeling stellar atmospheres (Carlsson et al. 1994; Barklem et al. 2003; Belyaev \& Barklem 2003).

To get a first overview of the possible non-adiabatic effects in lithium-helium ion-atom interactions at different excitation levels, a model approach (Belyaev \& Lebedev 2011; Belyaev 2013a) is applied here to estimate the cross-sections and rate coefficients in the corresponding non-radiative inelastic collisions.

\section{Nonadiabatic nuclear dynamics}

The theoretical treatment is performed within the standard adiabatic Born-Oppenheimer (BO) approach, which considers a collision problem in two steps: (i) electronic structure calculations and (ii) a study of the non-adiabatic nuclear dynamics.

Adiabatic BO potentials and electric dipole moment functions for several low-lying excited ${ }^{1,3} \Sigma^{+}$and ${ }^{1,3} \Pi$ states of the $\mathrm{LiHe}^{+}$ion were recently determined on a high-accuracy level of ab initio theory (Soldán \& Kraemer 2012). For the $\mathrm{X}^{1} \Sigma^{+}$ground state, the calculated characteristic spectroscopic data were found to be in perfect agreement with the best obtained results available from the literature in the past (Senff \& Burton 1986; Elford et al. 1999). For the ground state (X-state) and the first excited ${ }^{1,3} \Sigma^{+}$states (A- and a-states), radiative association processes were investigated in detail (Augustovičová et al. 2012; Bovino et al. 2011). In addition, depopulation of metastable $\mathrm{He}\left(2{ }^{3} \mathrm{~S}\right)$ 
Table 1. Parameters of the non-adiabatic regions for $\mathrm{LiHe}^{+}$.

\begin{tabular}{|c|c|c|c|c|c|c|}
\hline $\begin{array}{l}\text { Collision } \\
\text { channel } j\end{array}$ & $\begin{array}{l}\text { Molecular } \\
\text { state } j\end{array}$ & $\begin{array}{l}\text { Collision } \\
\text { channel } k\end{array}$ & $\begin{array}{l}\text { Molecular } \\
\text { state } k\end{array}$ & $\begin{array}{l}\text { Center } \\
R_{\mathrm{c}}(\mathrm{au})\end{array}$ & $\begin{array}{r}\text { Minimal splitting } \\
Z_{\mathrm{jk}}(\mathrm{au})\end{array}$ & $\begin{array}{r}\text { LZ parameter } \\
\xi_{\mathrm{jk}}(\mathrm{au})\end{array}$ \\
\hline \multicolumn{7}{|c|}{${ }^{1} \Sigma^{+}$symmetry } \\
\hline $\mathrm{Li}^{+}+\mathrm{He}\left(1 \mathrm{~s}^{2}{ }^{1} \mathrm{~S}\right)$ & $\mathrm{X}^{1} \Sigma^{+}$ & $\mathrm{Li}(2 \mathrm{~s})+\mathrm{He}^{+}$ & $\mathrm{A}^{1} \Sigma^{+}$ & 6.940 & $6.812(-1)$ & $1.157(1)$ \\
\hline $\mathrm{Li}(2 \mathrm{~s})+\mathrm{He}^{+}$ & $\mathrm{A}^{1} \Sigma^{+}$ & $\mathrm{Li}^{+}+\mathrm{He}\left(2 \mathrm{~s}^{1} \mathrm{~S}\right)$ & $\mathrm{B}^{1} \Sigma^{+}$ & 13.376 & $4.403(-2)$ & $4.137(-1)$ \\
\hline $\mathrm{Li}(2 \mathrm{~s})+\mathrm{He}^{+}$ & $\mathrm{A}^{1} \Sigma^{+}$ & $\mathrm{Li}^{+}+\mathrm{He}\left(2 \mathrm{~s}^{1} \mathrm{~S}\right)$ & $\mathrm{B}^{1} \Sigma^{+}$ & 2.728 & $9.589(-2)$ & $2.084(-1)$ \\
\hline $\mathrm{Li}^{+}+\mathrm{He}\left(2 \mathrm{~s}{ }^{1} \mathrm{~S}\right)$ & $\mathrm{B}^{1} \Sigma^{+}$ & $\mathrm{Li}(2 \mathrm{p})+\mathrm{He}^{+}$ & $\mathrm{C}^{1} \Sigma^{+}$ & 3.503 & $6.310(-3)$ & $2.040(-3)$ \\
\hline $\mathrm{Li}(2 \mathrm{p})+\mathrm{He}^{+}$ & $\mathrm{C}^{1} \Sigma^{+}$ & $\mathrm{Li}^{+}+\mathrm{He}\left(2 \mathrm{p}^{1} \mathrm{P}\right)$ & $\mathrm{D}^{1} \Sigma^{+}$ & 1.194 & $6.916(-3)$ & $6.387(-4)$ \\
\hline \multicolumn{7}{|c|}{${ }^{3} \Sigma^{+}$symmetry } \\
\hline $\mathrm{Li}(2 \mathrm{~s})+\mathrm{He}^{+}$ & $\mathrm{a}^{3} \Sigma^{+}$ & $\mathrm{Li}^{+}+\mathrm{He}\left(2 \mathrm{~s}{ }^{3} \mathrm{~S}\right)$ & $\mathrm{b}^{3} \Sigma^{+}$ & 15.511 & $2.180(-2)$ & $3.131(-1)$ \\
\hline $\mathrm{Li}(2 \mathrm{~s})+\mathrm{He}^{+}$ & $\mathrm{a}^{3} \Sigma^{+}$ & $\mathrm{Li}^{+}+\mathrm{He}\left(2 \mathrm{~s}{ }^{3} \mathrm{~S}\right)$ & $\mathrm{b}^{3} \Sigma^{+}$ & 2.448 & $9.488(-2)$ & $1.570(-1)$ \\
\hline $\mathrm{Li}^{+}+\mathrm{He}\left(2 \mathrm{~s}^{3} \mathrm{~S}\right)$ & $\mathrm{b}^{3} \Sigma^{+}$ & $\mathrm{Li}^{+}+\mathrm{He}\left(2 \mathrm{p}{ }^{3} \mathrm{P}\right)$ & $\mathrm{c}^{3} \Sigma^{+}$ & 15.089 & $3.913(-2)$ & $6.858(-1)$ \\
\hline $\mathrm{Li}^{+}+\mathrm{He}\left(2 \mathrm{~s}{ }^{3} \mathrm{~S}\right)$ & $\mathrm{b}^{3} \Sigma^{+}$ & $\mathrm{Li}^{+}+\mathrm{He}\left(2 \mathrm{p}{ }^{3} \mathrm{P}\right)$ & $\mathrm{c}^{3} \Sigma^{+}$ & 3.962 & $1.728(-3)$ & $4.326(-4)$ \\
\hline $\mathrm{Li}^{+}+\mathrm{He}\left(2 \mathrm{p}^{3} \mathrm{P}\right)$ & $\mathrm{c}^{3} \Sigma^{+}$ & $\mathrm{Li}(2 \mathrm{p})+\mathrm{He}^{+}$ & $\mathrm{d}^{3} \Sigma^{+}$ & 24.753 & $2.633(-3)$ & $3.709(-2)$ \\
\hline $\mathrm{Li}^{+}+\mathrm{He}\left(2 \mathrm{p}^{3} \mathrm{P}\right)$ & $\mathrm{c}^{3} \Sigma^{+}$ & $\mathrm{Li}(2 \mathrm{p})+\mathrm{He}^{+}$ & $\mathrm{d}^{3} \Sigma^{+}$ & 1.201 & $1.010(-2)$ & $3.041(-3)$ \\
\hline \multicolumn{7}{|c|}{${ }^{3} \Pi$ symmetry } \\
\hline $\mathrm{Li}^{+}+\mathrm{He}\left(2 \mathrm{p}^{3} \mathrm{P}\right)$ & $1^{3} \Pi$ & $\mathrm{Li}(2 \mathrm{p})+\mathrm{He}^{+}$ & $2{ }^{3} \Pi$ & 22.898 & $2.913(-3)$ & $1.899(-1)$ \\
\hline
\end{tabular}

Notes. $x(y) \equiv x \times 10^{y}$.

by radiative association processes was most recently studied (Augustovičová et al. 2014).

The excited-states adiabatic BO potentials calculated by Soldán \& Kraemer (2012) are used here as a basis for the present model calculations.

In general, in order to study non-adiabatic nuclear dynamics entirely by means of quantum mechanical methods one needs to know not only adiabatic BO potentials, but also nonadiabatic couplings. On the other hand, non-adiabatic transition probabilities, inelastic cross-sections, and rate coefficients can be estimated within a model approach, especially when complete quantum-chemical information is not available. Such a model approach, the so-called branching probability current method, that requires information only about adiabatic potentials, has been recently proposed (Belyaev 2013a) and applied to inelastic aluminium-hydrogen collisions (Belyaev 2013a,b). The main assumption of the approach is that non-adiabatic transition probabilities can be estimated within the framework of the Landau-Zener (LZ) model (Landau 1932a,b; Zener 1932). The bottleneck of the LZ-model application is twofold: (i) the conventional LZ formula requires the knowledge of diabatic parameters though a diabatization procedure is not uniquely defined; and (ii) usually, there are several non-adiabatic regions that determine the non-adiabatic nuclear dynamics without a particular order. The branching probability current method handles the latter by branching all incoming and outgoing probability currents after each traverse of the non-adiabatic regions in any order they appear during a collision. The method is similar to the branching classical trajectory method (Belyaev \& Lebedev 2011), which is based on classical trajectories and determines LZ parameters along each trajectory. The branching probability current method determines LZ parameters beforehand and then varies an internuclear distance $R$ without calculating any classical trajectories (see Belyaev 2013a).

The key point of the branching probability current method is a novel formula for a non-adiabatic transition probability within the LZ model, the so-called adiabatic-potential-based formula (Belyaev \& Lebedev 2011; Belyaev 2013a). Positions of non-adiabatic regions $R_{\mathrm{c}}$ are determined by minima of adiabatic splittings $Z_{\mathrm{jk}}=\left|U_{j}-U_{k}\right|$ between adjacent adiabatic states with $U_{\mathrm{j}, \mathrm{k}}(R)$ being adiabatic potentials. In these regions, the LZ parameters are determined by the following formula (Belyaev \& Lebedev 2011; Belyaev 2013a):

$\xi_{\mathrm{jk}}=\left.\frac{\pi}{2 \hbar} \sqrt{\frac{Z_{\mathrm{jk}}^{3}}{Z_{\mathrm{jk}}^{\prime \prime}}}\right|_{R=R_{\mathrm{c}}}$.

Primed quantities are referred to derivatives with respect to the internuclear distance $R$. Equation (1) expresses the LZ parameter only in terms of the adiabatic splitting $Z_{\mathrm{jk}}$ and its second distance derivative at $R_{\mathrm{c}}$. Finally, a non-adiabatic transition probability $p_{\mathrm{jk}}^{\mathrm{LZ}}$ after a single traverse of a non-adiabatic region is written in the usual form,

$p_{\mathrm{jk}}^{\mathrm{LZ}}=\exp \left(-\xi_{\mathrm{jk}} / v\right)$

where $v$ is a radial velocity of colliding atoms. All values are evaluated at the centers of the non-adiabatic regions.

Thus, Eqs. (1) and (2) allow one to calculate non-adiabatic transition probabilities in each non-adiabatic region based on the information about adiabatic potentials only. Then branching of incoming and outgoing currents allows one to compute probabilities for transitions between a given initial state and a given final state, and finally inelastic cross-sections and rate coefficients.

\section{Results of calculations}

\subsection{Nonadiabatic regions}

In the present paper, we treat non-adiabatic transitions separately between the low-lying ${ }^{1} \Sigma^{+}$states, between the low-lying ${ }^{3} \Sigma^{+}$states, and between the pair of the lowest excited ${ }^{3} \Pi$ states. The adiabatic BO potentials for these symmetries are taken from Soldán \& Kraemer (2012). For the lowest excited pair of ${ }^{1} \Pi$ states, the form and relative separation of their potentials (Soldán \& Kraemer 2012) indicate that non-adiabatic coupling between them is negligible. It is therefore not considered here. Transitions between states of different symmetries are also not considered in the present estimates.

The parameters of the non-adiabatic regions for the ${ }^{1} \Sigma^{+},{ }^{3} \Sigma^{+}$ and ${ }^{3} \Pi$ symmetries of $\mathrm{LiHe}^{+}$are collected in Table 1 . The centers $R_{\mathrm{c}}$ of these regions are determined by the local minima of 
A. K. Belyaev et al.: Non-radiative inelastic processes in lithium-helium ion-atom collisions

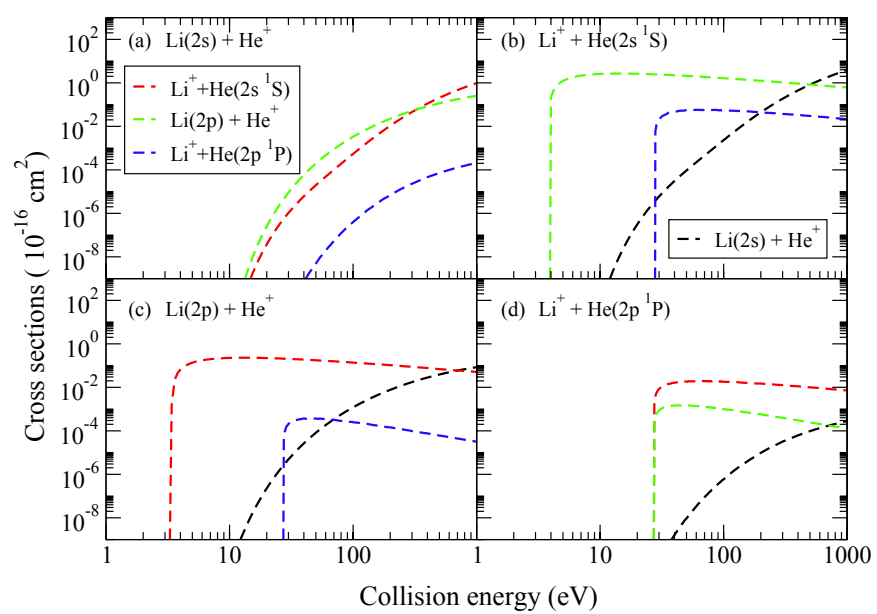

Fig. 1. Inelastic cross-sections within the ${ }^{1} \Sigma^{+}$system. The initial collision channel is indicated in each panel. The key for final channels is common to all panels and is shown in panels a) and b). The statistical probability factors for the initial channels are taken into account in the cross-sections.

the corresponding adiabatic splittings $Z_{\mathrm{jk}}$. The $\mathrm{LZ}$ parameters $\xi_{\mathrm{jk}}$ are then calculated in each region by means of Eq. (1). There are five non-adiabatic regions within the ${ }^{1} \Sigma^{+}$symmetry, six regions within the ${ }^{3} \Sigma^{+}$symmetry, and one region within the ${ }^{3} \Pi$ symmetry. Table 1 also shows that within the ${ }^{1} \Sigma^{+}$and ${ }^{3} \Sigma^{+}$symmetries, several non-adiabatic regions can exist for certain pairs of adjacent potentials, which makes the non-adiabatic nuclear dynamics more complicated. Actually, the branching probability current method applied here takes this situation automatically into account. The calculated LZ parameters allow us to estimate inelastic cross-sections and rate coefficients.

\subsection{Inelastic cross-sections}

According to the data for non-adiabatic transitions within the ${ }^{1} \Sigma^{+}$symmetry, that are listed in Table 1 , a non-adiabatic region around $R_{\mathrm{c}}=6.94$ au is indicated by the present model calculations for the adiabatic potentials of the ground state $\left(\mathrm{X}^{1} \Sigma^{+}\right)$and the first excited state $\left(\mathrm{A}^{1} \Sigma^{+}\right)$. The energy gap, however, between these two potentials is very big (see Table 1 for the minimum of the adiabatic splitting $Z_{\mathrm{jk}}$ ), resulting in the large LZ parameter $\xi_{\mathrm{jk}}$ and making the probabilities for non-adiabatic transitions from and to the ground molecular state extremely small. Inelastic non-radiative collision processes involving the $\mathrm{Li}^{+}+\mathrm{He}\left(1 \mathrm{~s}^{2}{ }^{1} \mathrm{~S}\right)$ channel (both as the initial and as the final channels) have, thus, negligible inelastic cross-sections and rate coefficients and are therefore disregarded here.

The inelastic cross-sections for the other transitions within the ${ }^{1} \Sigma^{+}$symmetry are shown in Fig. 1 as a function of the collision energy $E$. The initial collision channel is specified in the top of each panel, whereas the final collision channels, which are labeled by colors, are defined in the insets of panels (a) and (b). The definitions of the color labels are common for all figures in the present paper.

Figure 1 shows that practically all partial cross-sections have large energy thresholds, reaching from $3.4 \mathrm{eV}$ up to about $28 \mathrm{eV}$. These large thresholds are due to the fact that most non-adiabatic regions within the ${ }^{1} \Sigma^{+}$symmetry are located at short internuclear distances in the repulsive part of the adiabatic potentials with $R_{\mathrm{c}}<3.5$ au. For non-adiabatic transitions to occur, the collision system has therefore to have enough kinetic energy. For

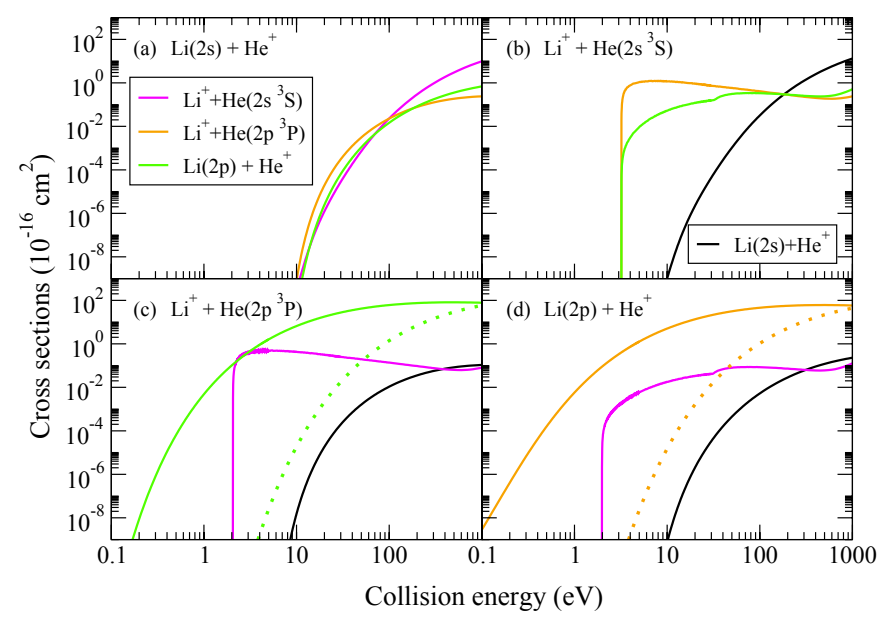

Fig. 2. Inelastic cross-sections within the ${ }^{3} \Sigma^{+}$system (solid lines) and within the ${ }^{3} \Pi$ system (dotted lines). The initial collisional channel is indicated in each panel. The key for final channels is common to all panels and is shown in panels a) and b). The statistical probability factors for the initial channels are taken into account in the cross-sections.

the charge transfer reaction $\mathrm{Li}(2 \mathrm{~s})+\mathrm{He}^{+} \rightleftharpoons \mathrm{Li}^{+}+\mathrm{He}\left(2 \mathrm{~s}{ }^{1} \mathrm{~S}\right)$, however, there exists a second non-adiabatic region at large internuclear distance $\left(R_{\mathrm{c}}=13.376 \mathrm{au}\right)$. The LZ parameter here is relatively large and the non-adiabatic transition probabilities get remarkable values at rather high collision energies.

At low collision energies, most partial cross-sections have rather small values in Fig. 1. An exception here is the $\mathrm{Li}^{+}+$ $\mathrm{He}\left(2 \mathrm{~s}^{1} \mathrm{~S}\right) \rightleftharpoons \mathrm{Li}(2 \mathrm{p})+\mathrm{He}^{+}$charge transfer into excited state processes. For this endothermic process, the cross-section increases rapidly right above the threshold and reaches its maximum value of $2.7 \AA^{2}$ at a collision energy of $E=14 \mathrm{eV}$. Similar large crosssections are obtained for other processes only at higher collision energies.

The calculated partial inelastic cross-sections within the ${ }^{3} \Sigma^{+}$ system and within the ${ }^{3} \Pi$ system are shown in Fig. 2 by solid and dotted lines, respectively. Initial and final collisional channels are specified here in the same way as in Fig. 1. Obviously many partial cross-sections have also relatively large energy thresholds for the same reasons discussed before for the ${ }^{1} \Sigma^{+}$system. The cross-sections for the $\mathrm{Li}^{+}+\mathrm{He}\left(2 \mathrm{p}^{3} \mathrm{P}\right) \rightleftharpoons \mathrm{Li}(2 \mathrm{p})+\mathrm{He}^{+}$charge transfer into excited state processes, on the other hand, have a small energy threshold (actually, $0.08 \mathrm{eV}$ for the endothermic process and practically no energy threshold for the exothermic process). This occurs because the second non-adiabatic region at large internuclear distance with $R_{\mathrm{c}}=24.753 \mathrm{au}$ is located between the $\mathrm{c}^{3} \Sigma^{+}$and $\mathrm{d}^{3} \Sigma^{+}$molecular states with the closelying potentials in this region (Soldán \& Kraemer 2012). For the ${ }^{3} \Pi$ system the non-adiabatic region also occurs at a long distance, $R_{\mathrm{c}}=22.898 \mathrm{au}$. However, the LZ parameter obtained in this region appears to be large, given the effect that the nonadiabatic transitions with observable probabilities and larger inelastic cross-sections occur only at higher energies (indicated by the dotted lines in Fig. 2).

Cross-sections obtained here for the ${ }^{3} \Sigma^{+}$system have typically larger values compared to those for the ${ }^{1} \Sigma^{+}$system. The values range from a few $\AA^{2}$ at low collision energies to 5-7 $\AA^{2}$ at $E=10 \mathrm{eV}$ and up to $80 \AA^{2}$ at high energies for the $\mathrm{Li}^{+}+\mathrm{He}\left(2 \mathrm{p}^{3} \mathrm{P}\right) \rightleftharpoons \mathrm{Li}(2 \mathrm{p})+\mathrm{He}^{+}$charge transfer processes. For these processes, the transitions mainly take place in the non-adiabatic region around $R=24.753 \mathrm{au}$, that is, at large internuclear distances. In addition, the excitation/de-excitation 


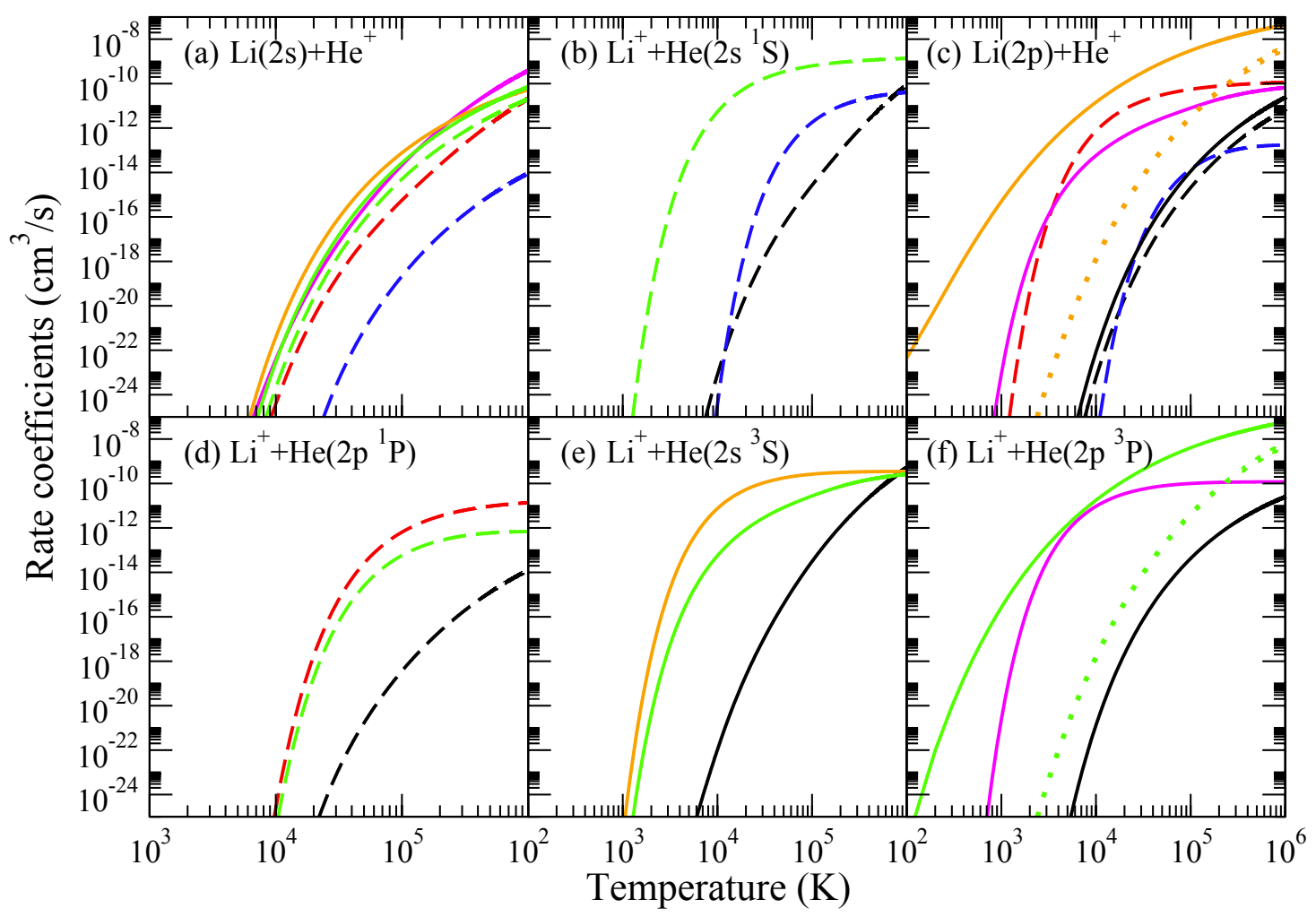

Fig. 3. Inelastic rate coefficients within the ${ }^{1} \Sigma^{+}$(dashed lines), ${ }^{3} \Sigma^{+}$(solid lines), and ${ }^{3} \Pi$ (dotted lines) systems. The initial collisional channel is indicated in each panel. The key for final channels is common to all panels and is labeled by colors: black lines correspond to $\mathrm{Li}(2 \mathrm{~s})+\mathrm{He}{ }^{+}$; red lines to $\mathrm{Li}^{+}+\mathrm{He}\left(2 \mathrm{~s}{ }^{1} \mathrm{~S}\right)$; green to $\mathrm{Li}(2 \mathrm{p})+\mathrm{He}^{+}$; blue to $\mathrm{Li}^{+}+\mathrm{He}\left(2 \mathrm{p}{ }^{1} \mathrm{P}\right)$; magenta to $\mathrm{Li}^{+}+\mathrm{He}\left(2 \mathrm{~s}^{3} \mathrm{~S}\right)$; and orange to $\mathrm{Li}^{+}+\mathrm{He}\left(2 \mathrm{p}{ }^{3} \mathrm{P}\right)$.

processes $\mathrm{Li}^{+}+\mathrm{He}\left(2 \mathrm{~s}^{3} \mathrm{~S}\right) \rightleftharpoons \mathrm{Li}^{+}+\mathrm{He}\left(2 \mathrm{p}{ }^{3} \mathrm{P}\right)$ also have large cross-section values reaching $1.2 \AA^{2}$ as a maximum.

Apart from the processes discussed here, other partial processes can occur via non-adiabatic transitions within different molecular symmetries. The $\mathrm{Li}^{+}+\mathrm{He}\left(2 \mathrm{p}{ }^{3} \mathrm{P}\right) \rightleftharpoons \mathrm{Li}(2 \mathrm{p})+\mathrm{He}^{+}$ charge transfer processes, for example, involve transitions within the ${ }^{3} \Sigma^{+}$and ${ }^{3} \Pi$ systems and the $\mathrm{Li}(2 \mathrm{~s})+\mathrm{He}^{+} \rightleftharpoons \mathrm{Li}(2 \mathrm{p})+\mathrm{He}^{+}$ excitation/de-excitation processes are based on non-adiabatic transitions within the ${ }^{1} \Sigma^{+}$and ${ }^{3} \Sigma^{+}$systems.

Finally, it should be emphasized that the LZ model might provide less accurate transition probabilities for non-adiabatic regions with large adiabatic splittings or for cases when other quantum effects, such as tunneling, are substantial. In those situations the model estimates have larger uncertainties for small transition probabilities and, hence, low cross-sections. A fully quantum-mechanical treatment of the tunneling effect at short internuclear distances in the repulsive part of the potentials, for instance, could increase some non-adiabatic transition probabilities and lower the energy threshold. This would eventually increase the rate coefficients, whereas the model provides smaller estimates. However, for cases with substantial transition probabilities, the model applied here has been found to provide reliable estimates of the dominant cross-sections and rate coefficients (Belyaev 2013a).

\subsection{Rate coefficients}

The calculated inelastic rate coefficients are presented in Fig. 3 as a function of the temperature $T$ in the range between $10^{3}$ to $10^{6} \mathrm{~K}$. The initial collision channels are again specified in the top of each panel and the same color labels are used for the final collision channels as in Figs. 1 and 2.
The gross behavior of the rate coefficients reflects the characteristics of the corresponding cross-sections. The rate coefficients increase from very low values at low temperatures up to roughly $6 \times 10^{-8} \mathrm{~cm}^{3} / \mathrm{s}$ at high temperatures. Low rate coefficients for the partial processes at low temperatures are partly due to the large energy thresholds for the partial cross-sections and partly to low values of the low-energy cross-sections, as discussed above. The high temperature increase of the rate coefficients corresponds to the increase of the cross-sections at high collision energies.

It is seen from Fig. 3 that depopulation of the $\mathrm{Li}(2 \mathrm{~s})+\mathrm{He}^{+}$ channel is inefficient at low temperatures that are below $10^{4} \mathrm{~K}$. The partial rate coefficients are smaller than $4 \times$ $10^{-22} \mathrm{~cm}^{3} / \mathrm{s}$, and all depopulation processes are endothermic, since the cross-section for the only exothermic channel, $\mathrm{Li}^{+}+\mathrm{He}\left(1 \mathrm{~s}^{2}{ }^{1} \mathrm{~S}\right)$, is negligible. At high temperatures, depopulation of the $\mathrm{Li}(2 \mathrm{~s})+\mathrm{He}^{+}$channel can occur due to several processes, which are mostly due to charge transfer and excitation. The rate coefficients can reach the values up to $5 \times 10^{-10} \mathrm{~cm}^{3} / \mathrm{s}$, which are mainly due to transitions into the $\mathrm{Li}^{+}+\mathrm{He}\left(2 \mathrm{~s}{ }^{3} \mathrm{~S}\right)$ final channel. Depopulation of the $\mathrm{Li}^{+}+\mathrm{He}\left(2 \mathrm{p}{ }^{1} \mathrm{P}\right)$ channel is much less efficient than that for the $\mathrm{Li}(2 \mathrm{~s})+\mathrm{He}^{+}$channel. Actually it is the least efficient among the treated collision channels. Depopulation of the $\mathrm{Li}^{+}+\mathrm{He}\left(2 \mathrm{~s}{ }^{1} \mathrm{~S}\right)$ channel is more efficient even at low temperatures mainly because of the $\mathrm{Li}^{+}+\mathrm{He}\left(2 \mathrm{~s}^{1} \mathrm{~S}\right) \rightarrow \mathrm{Li}(2 \mathrm{p})+\mathrm{He}^{+}$charge transfer into excited state process. The corresponding rate coefficient is equal to $5.4 \times 10^{-12} \mathrm{~cm}^{3} / \mathrm{s}$ at $T=10^{4} \mathrm{~K}$ and increases up to $1.4 \times 10^{-9} \mathrm{~cm}^{3} / \mathrm{s}$ at $T=10^{6} \mathrm{~K}$. The efficiency of decay of the $\mathrm{Li}^{+}+\mathrm{He}\left(2 \mathrm{~s}{ }^{3} \mathrm{~S}\right)$ channel is comparable to that of the $\mathrm{Li}^{+}+\mathrm{He}\left(2 \mathrm{~s}{ }^{1} \mathrm{~S}\right)$. The rate coefficient equals $7.5 \times 10^{-12} \mathrm{~cm}^{3} / \mathrm{s}$ for the $\mathrm{Li}^{+}+\mathrm{He}\left(2 \mathrm{~s}^{3} \mathrm{~S}\right) \rightarrow \mathrm{Li}^{+}+\mathrm{He}\left(2 \mathrm{p}^{3} \mathrm{P}\right)$ excitation process 
at $T=10^{4} \mathrm{~K}$ and is $5.2 \times 10^{-10} \mathrm{~cm}^{3} / \mathrm{s}, 3.5 \times 10^{-10} \mathrm{~cm}^{3} / \mathrm{s}$, and $2.6 \times 10^{-10} \mathrm{~cm}^{3} / \mathrm{s}$ for the $\mathrm{Li}(2 \mathrm{~s})+\mathrm{He}^{+}$, the $\mathrm{Li}^{+}+\mathrm{He}\left(2 \mathrm{p}^{3} \mathrm{P}\right)$, and the $\mathrm{Li}(2 \mathrm{p})+\mathrm{He}^{+}$final channels, respectively, at $T=10^{6} \mathrm{~K}$. The largest values for the rate coefficients correspond to the $\mathrm{Li}^{+}+\mathrm{He}\left(2 \mathrm{p}^{3} \mathrm{P}\right) \rightleftharpoons \mathrm{Li}(2 \mathrm{p})+\mathrm{He}^{+}$charge transfer processes. They are equal to $1.8 \times 10^{-11} \mathrm{~cm}^{3} / \mathrm{s}$ and $1.5 \times 10^{-11} \mathrm{~cm}^{3} / \mathrm{s}$ for the direct and the inverse processes, respectively, at $T=10^{4} \mathrm{~K}$, and to $5.8 \times 10^{-8} \mathrm{~cm}^{3} / \mathrm{s}$ and $4.4 \times 10^{-8} \mathrm{~cm}^{3} / \mathrm{s}$ for direct and inverse processes, respectively, at $T=10^{6} \mathrm{~K}$. The rate coefficients for these charge transfer processes in the ${ }^{3} \Pi$ symmetry also reach values at $T=10^{6} \mathrm{~K}$, which are almost exactly one order of magnitude smaller than those for the ${ }^{3} \Sigma$ system, as shown by the dotted curves in Fig. 3. At relatively low temperatures, the $\mathrm{Li}^{+}+\mathrm{He}\left(2 \mathrm{p}^{3} \mathrm{P}\right) \rightarrow \mathrm{Li}^{+}+\mathrm{He}\left(2 \mathrm{~s}^{3} \mathrm{~S}\right)$ de-excitation process also contributes noticeably into decay of $\mathrm{He}\left(2 \mathrm{p}{ }^{3} \mathrm{P}\right)$ in collisions with $\mathrm{Li}^{+}$: the corresponding rate coefficient equals $9.4 \times 10^{-12} \mathrm{~cm}^{3} / \mathrm{s}$ at $T=10^{4} \mathrm{~K}$.

All partial inelastic rate coefficients decrease rapidly with decreasing the temperature below $10^{4} \mathrm{~K}$. The only noticeable rate coefficient remaining at low temperatures is that for the exothermic charge transfer process $\mathrm{Li}(2 \mathrm{p})+\mathrm{He}^{+} \rightarrow \mathrm{Li}^{+}+\mathrm{He}\left(2 \mathrm{p}^{3} \mathrm{P}\right)$.

\section{Discussion and conclusion}

The non-radiative cross-sections and rate coefficients estimated in the present paper can be compared to the radiative association cross-sections and rate coefficients calculated by Augustovičová et al. $(2012,2014)$ for the same collision systems. The comparison shows that there are a couple of basic differences some of which are listed below.

First of all, the radiative association treatment provides noticeable cross-sections and rate coefficients from and to the ground molecular state $\mathrm{LiHe}^{+}\left(\mathrm{X}^{1} \Sigma^{+}\right)$, for which the present model study estimates negligible non-radiative cross-sections and rate coefficients. The non-radiative processes, thus, do not change depopulation of the $\mathrm{Li}^{+}+\mathrm{He}\left(1 \mathrm{~s}^{2}{ }^{1} \mathrm{~S}\right)$ channel.

Second, the energy dependence of the cross-sections and the temperature dependence of the rate coefficients for radiative and non-radiative processes are totally different. The radiative cross-sections and rate coefficients generally decrease with increasing collision energy and temperature in the low-energy regime. In contrast to this, an increase of the cross-sections and rate coefficients with increasing energy and temperature is observed for non-radiative processes. A general conclusion from this controversial behavior would be that radiative processes are expected to be dominant over non-radiative ones at low energies. This finding actually agrees with the results of the treatment of radiative and non-radiative processes in $\mathrm{He}^{+}+\mathrm{H}$ collisions (Zygelman et al. 1989), as well as in cold and ultra-cold $\mathrm{Yb}^{+}+\mathrm{Rb}$ collisions (Sayfutyarova et al. 2013).

Third, depopulation of the $\mathrm{Li}(2 \mathrm{~s})+\mathrm{He}^{+}$collision system due to the radiative association into both the ${ }^{1} \Sigma^{+}$and the ${ }^{3} \Sigma^{+}$symmetries, i.e., depopulation of the A and a molecular states (see Table 1), was recently studied by Augustovičová et al. (2012). It is shown there that the dominant channel corresponds to the $\mathrm{A} \rightarrow$ $X$ transitions with a rate coefficient varying from $10^{-14} \mathrm{~cm}^{3} / \mathrm{s}$ at $0.1 \mathrm{~K}$ down to $10^{-16} \mathrm{~cm}^{3} / \mathrm{s}$ at $10^{4} \mathrm{~K}$. In this temperature range the present estimates show that the non-radiative depopulation of the same states has a maximum total rate coefficient of $4.4 \times 10^{-22} \mathrm{~cm}^{3} / \mathrm{s}$ at $10^{4} \mathrm{~K}$ due to transitions a $\rightarrow \mathrm{c}, \mathrm{b}, \mathrm{d}$ within ${ }^{3} \Sigma^{+}$symmetry, whereas the rate coefficients for depopulation of the A state within the ${ }^{1} \Sigma^{+}$symmetry are much smaller than in the ${ }^{3} \Sigma^{+}$symmetry (see Fig. 3). This leads to the conclusion that the radiative depopulation of the $\mathrm{Li}(2 \mathrm{~s})+\mathrm{He}^{+}$collision system dominates over the non-radiative depopulation in the lowtemperature range (below $10^{4} \mathrm{~K}$ ).

Fourth, most recently, depopulation of the $\mathrm{Li}^{+}+\mathrm{He}\left(2 \mathrm{~s}{ }^{3} \mathrm{~S}\right)$ collision system due to radiative association in the ${ }^{3} \Sigma^{+}$symmetry, i.e. depopulation of the $b$ molecular state, was studied by Augustovičová et al. (2014). The dominant transition here is $b \rightarrow a$ with the corresponding rate coefficient of $4.54 \times$ $10^{-15} \mathrm{~cm}^{3} / \mathrm{s}$ at $10^{4} \mathrm{~K}$. For the same transition, the non-radiative rate coefficient is much smaller, $10^{-21} \mathrm{~cm}^{3} / \mathrm{s}$ at $10^{4} \mathrm{~K}$. On the other hand, according to Fig. 3, the rate coefficients for depopulation of the $b$ state into other channels are much larger: $7.5 \times 10^{-12} \mathrm{~cm}^{3} / \mathrm{s}$ at $10^{4} \mathrm{~K}$ for the $\mathrm{b} \rightarrow \mathrm{c}$ transition (i.e., the $\mathrm{Li}^{+}+\mathrm{He}\left(2 \mathrm{~s}^{3} \mathrm{~S}\right) \rightarrow \mathrm{Li}^{+}+\mathrm{He}\left(2 \mathrm{p}^{3} \mathrm{P}\right)$ excitation process $)$, and $5.6 \times 10^{-14} \mathrm{~cm}^{3} / \mathrm{s}$ at $10^{4} \mathrm{~K}$ for the $\mathrm{b} \rightarrow \mathrm{d}$ transition (i.e., the $\mathrm{Li}^{+}+\mathrm{He}\left(2 \mathrm{~s}^{3} \mathrm{~S}\right) \rightarrow \mathrm{Li}(2 \mathrm{p})+\mathrm{He}^{+}$charge transfer process $)$. Comparison of the temperature dependence of the rate coefficients for the radiative and non-radiative depopulation shows that the non-radiative depopulation dominates over the radiative one for the $\mathrm{Li}^{+}+\mathrm{He}\left(2 \mathrm{~s}{ }^{3} \mathrm{~S}\right)$ collision channel at $T>3000 \mathrm{~K}$.

Based on these results, it can be concluded that at relatively high temperatures a non-radiative decay (due to non-adiabatic transitions) is expected to dominate over a radiative decay for higher-lying excited states. A critical temperature has to be determined in each particular case. More generally, it appears that radiative and non-radiative processes apply to different areas in interstellar environments when studying atomic or molecular astrophysical questions. For example, non-radiative collision processes can, therefore, be expected to be dominant in stellar atmospheres.

Acknowledgements. A.K.B. gratefully acknowledges support from the Russian Foundation for Basic Research (Grant No. 13-03-00163-a) and from the MaxPlanck Institute for Astrophysics at Garching (Germany). L.A. acknowledges funding from the Grant Agency of the Charles University in Prague - GAUK (Grant No. 550112).

\section{References}

Augustovičová, L., Špirko, V., Kraemer, W. P., \& Soldán, P. 2012, Chem. Phys. Lett., 531, 59

Augustovičová, L., Kraemer, W. P., \& Soldán, P. 2014, ApJ, 782, 46

Barklem, P. S., Belyaev, A. K., \& Asplund, M. 2003, A\&A, 409, L1

Belyaev, A. K. 2013a, Phys. Rev. A, 88, 2704

Belyaev, A. K. 2013b, A\&A, 560, A60

Belyaev, A. K., \& Barklem, P. S. 2003, Phys. Rev. A, 68, 2703

Belyaev, A. K., \& Lebedev, O. V. 2011, Phys. Rev. A, 84, 4701

Bovino, S., Tacconi, M., \& Gianturco, F. A. 2011, ApJ, 740, 101, erratum 2012, ApJ, 748, 150

Burles, S., Nollett, K. M., \& Turner, M. S. 2001, ApJ, 552, L1

Carlsson, M., Rutten, R. J., Bruls, J. H. M. J., \& Shchukina, N. G. 1994, A\&A, 288,860

Elford, M. T., Røeggen, I., \& Skullerud, H. R. 1999, J. Phys. B At. Mol. Phys., 32,1873

Galli, D., \& Palla, F. 1998, A\&A, 335, 403

Galli, D., \& Palla, F. 2013, ARA\&A, 51, 163

Landau, L. D. 1932a, Phys. Z. Sowietunion, 1, 88

Landau, L. D. 1932b, Phys. Z. Sowietunion, 2, 46

Loeb, A. 2001, ApJ, 555, L1

Palla, F., Galli, D., \& Silk, J. 1995, ApJ, 451, 44

Sayfutyarova, E. R., Buchachenko, A. A., Yakovleva, S. A., \& Belyaev, A. K. 2013, Phys. Rev. A, 87, 2717

Senff, U. E., \& Burton, P. G. 1986, Mol. Phys., 58, 637

Soldán, P., \& Kraemer, W. P. 2012, Chem. Phys., 393, 135

Stancil, P. C., \& Dalgarno, A. 1997, ApJ, 490, 76

Stancil, P. C., \& Zygelman, B. 1996, ApJ, 472, 102

Stancil, P. C., Lepp, S., \& Dalgarno, A. 1998, ApJ, 509, 1

Stancil, P. C., Loeb, A., Zaldarriaga, M., Dalgarno, A., \& Lepp, S. 2002, ApJ, 580,29

Zener, C. 1932, Proc. Roy. Soc. A, 137, 696

Zygelman, B., Dalgarno, A., Kimura, M., \& Lane, N. F. 1989, Phys. Rev. A, 40, 2340 